\title{
Análise do uso de medicamentos em idosos
}

\author{
Analysis of the use of medications in elderly \\ Análisis del uso de medicamentos en ancianos
}

Cristiane Ribeiro Maués ${ }^{1}$, Mariana Morgado Fernandez ${ }^{1}$, Quezia Prado Nunes ${ }^{1}$, Ana Caroline Coelho Gomes $^{1 *}$, Letícia Pinheiro Nascimento ${ }^{1}$, Ana Katarina Marques de Lima ${ }^{1}$, Stéphanie de Windson Cruz Navarro'.

\section{RESUMO}

Objetivo: Analisar o uso de medicamentos em idosos, avaliar a presença de polifarmácia e o padrão farmacológico. Métodos: Estudo transversal, realizado pela análise de prontuários em ambulatório de saúde do idoso no período de janeiro de 2014 até dezembro de 2016. Variáveis analisadas: padrão farmacológico desses pacientes, comorbidades existentes e polifarmácia. Os dados foram analisados utilizando-se a estatística descritiva e o teste Qui-quadrado de Aderência. Foi estabelecido o valor de $p<0,05$ para diferenças estatisticamente significativas com auxílio do programa BioEstat. Resultados: Foram analisados 236 prontuários, sendo $163(69,1 \% \%)$ de pacientes do gênero feminino e $73(30,9 \%)$ do gênero masculino. As doenças referidas na amostra foram $75,6 \%$ de hipertensão, 49,4\% diabetes e 16,2\% de distúrbios respiratórios. No padrão farmacológico, têm-se um predomínio do uso de anti-hipertensivos $(41,0 \%)$, de hipoglicemiantes orais $(10,5 \%)$ e de antiagregantes plaquetários $(5,9 \%)$. A polifarmácia é mais frequente em anciãos $(9,3 \%)$ do que em idosos $(7,6 \%)$. Conclusão: Encontrou-se maioria do sexo feminino. A principal doença encontrada foi a hipertensão arterial, seguida por diabetes e distúrbios respiratórios. Os antihipertensivos são os medicamentos mais prescritos, seguidos de hipoglicemiantes orais e antiagregantes plaquetários. Foi observada maior porcentagem de polifarmácia em anciãos quando comparados aos idosos.

Palavras Chave: Polifarmácia, Idosos, Ambulatorial.

\begin{abstract}
Objective: To analyze the use of medication in the elderly, evaluate the presence of polypharmacy and the pharmacological pattern. Methods: A cross-sectional study, carried out by the medical records of the elderly health clinic in the period from January 2014 to December 2016. Variables analyzed: pharmacological pattern of these patients, existing comorbidities and polypharmacy. The data were analyzed using descriptive statistics and the Chi-square test of Adherence. Results: A total of 236 medical records were analyzed, of which 163 $(69.1 \%)$ of female patients and $73(30.9 \%)$ of the patients were male gender. The diseases mentioned in the sample were $75.6 \%$ of hypertension, $49.4 \%$ of diabetes and $16.2 \%$ of respiratory disorders. In the pharmacological standard, antihypertensives $(41.0 \%)$, oral hypoglycemic agents $(10.5 \%)$ and antiplatelet agents $(5.9 \%)$ are predominant. Polypharmacy is more frequent in the elderly $(9.3 \%)$ than in the elderly $(7.6 \%)$. Conclusion: A majority of females were found. The main disease was hypertension, followed by diabetes and respiratory disorders. Antihypertensives are the most prescribed medications, followed by oral hypoglycemic agents and antiplatelet agents. A higher percentage of polypharmacy was observed in the elderly when compared to the elderly.
\end{abstract}

Key words: Polypharmacy, Seniors, Ambulatory.

${ }^{1}$ Centro Universitário do Estado do Pará (CESUPA), Belém-Pará. *E-mail: carolsscoelho@gmail.com 


\section{RESUMEN}

Objetivo: Analizar el uso de medicamentos en ancianos, evaluar la presencia de polifarmacia y el patrón farmacológico. Métodos: Estudio transversal, realizado por el análisis de prontuarios del ambulatorio de salud del anciano en el período de enero de 2014 hasta diciembre de 2016. Variables analizadas: patrón farmacológico de esos pacientes, comorbilidades existentes y polifarmacia. Los datos fueron analizados utilizando la estadística descriptiva y la prueba Qui-cuadrado de Adherencia. Se estableció el valor de $p<0,05$ para diferencias estadísticamente significativas con ayuda del programa BioEstat. Resultados: Se analizaron 236 prontuarios, siendo $163(69,1 \%)$ de pacientes del género femenino y $73(30,9 \%)$ del grupo género masculino. Las enfermedades mencionadas en la muestra fueron $75,6 \%$ de hipertensión, $49,4 \%$ de la diabetes y $16,2 \%$ de trastornos respiratorios. En el patrón farmacológico, se tiene un predominio del uso de antihipertensivos $(41,0 \%)$, de hipoglicemiantes orales $(10,5 \%)$ y de antiagregantes plaquetarios $(5,9 \%)$. La polifarmacia es más frecuente en ancianos $(9,3 \%)$ que en ancianos $(7,6 \%)$. Conclusión: Se ha encontrado la mayoría del sexo femenino. La principal enfermedad encontrada fue la hipertensión arterial, seguida por la diabetes y los trastornos respiratorios. Los antihipertensivos son los medicamentos más prescritos, seguidos de hipoglucemiantes orales y antiagregantes plaquetarios. Se observó un mayor porcentaje de polifarmacia en ancianos en comparación con los ancianos.

Palabras clave: Polifarmacia, Ancianos, Ambulatorios.

\section{INTRODUÇÃO}

A senescência é acompanhada de diversas modificações fisiológicas, pelas quais os diversos sistemas do organismo humano buscam continuar realizando suas funções, embora necessitem adaptar-se às alterações ocorridas naturalmente durante a vida. Como consequência de tais modificações, torna-se necessária a introdução de medicamentos para contornar as disfunções progressivas, objetivando assegurar uma melhor qualidade de vida. A partir dos 60 anos de idade, a polifarmácia e o uso de medicamentos inadequados continuam sendo problemas comuns, que se agravam nas idades mais avançadas e quanto piores forem as condições de saúde (CIOSAK SI, et al., 2011; PEREIRA LRL, et al., 2004; ARAÚJO ENP e LOPES RGC, 2010; MOSEGUI GBG, et al., 2005; ROZENFELD, 2003).

A análise dos dados demográficos demonstra que a população idosa tem se tornado cada vez mais numerosa em escala nacional e internacional. Alguns fatores que contribuíram para o processo de envelhecimento populacional são: queda da taxa de fecundidade, queda da mortalidade geral e aumento da esperança de vida ao nascer. Para isso, houve contribuição da melhoria nutricional, suporte proporcionado pelos avanços tecnológicos no campo da medicina, elevação dos níveis de higiene pessoal e ambiental, entre outros (CIOSAK SI, et al., 2011; ARAÚJO ENP e LOPES RGC, 2010; ACURCIO FE, et al., 2003; CORRER CJ, et al., 2007; MARIN MJS, et al., 2008).

No Brasil, há uma prevalência concomitante de doenças infectocontagiosas e crônico-degenerativas, criando um ambiente em que morbimortalidade ainda se encontra elevada, caracterizando uma transição prolongada com tríplice carga de doença. Dentro do território nacional, há polarização epidemiológica, visto que diferentes regiões possuem situações epidemiológicas contrastantes (SCHRAMM JMA, et al., 2004; FRENK J, et al., 1989).

Juntamente ao crescente número de idosos, cresce também de forma equitativa, o consumo de medicamentos por essa população, pois possivelmente, esta corresponde ao grupo etário mais medicalizado atualmente. A prescrição médica a essa parcela da população deve ser realizada de maneira bastante atenta e minuciosa, assim como a explicação da receita, para que o acompanhamento farmacológico associado às recomendações não-farmacológicas obtenha os resultados esperados e proporcione uma melhor qualidade de vida (ARAÚJO ENP e LOPES RGC, 2010; MOSEGUI GBG, et al., 1999; ROMANO-LIEBER NS, et al., 2002). 
A razão de se pesquisar a frequência da utilização de medicamentos por idosos é justificada pela importância do conhecimento de quais fármacos são mais utilizados, tendo em vista as diversas possibilidades de interações medicamentosas, que podem causar prejuízo ao efeito esperado para o tratamento instituído. Outro motivo que justifica a pesquisa é o alto índice de polifarmácia pelos idosos, devido a tendência atual da medicina setorizada, onde cada especialista realiza sua prescrição individualmente, muitas vezes sem conhecimento das medicações já prescritas antes para o mesmo paciente. Tais eventos aumentam a possibilidade de iatrogenias cometidas pelos médicos, pois idosos são pertencentes a um grupo de atenção especial no momento da adequação do tratamento farmacológico (ACURCIO FA, et al., 2009).

É necessário que seja observado onde ocorrem as falhas no tratamento do idoso, se ocorrem no momento da prescrição, na falta de acesso ao medicamento, na automedicação e/ou outros. A partir de então, é possível realizar propostas de intervenção para contornar a situação, através de uma atualização dos profissionais sobre os critérios de prescrição de medicamentos para a população idosa (CORRER CJ, et al., 2007).

Este estudo tem como objetivo analisar o uso de medicamentos em idosos atendidos no ambulatório de saúde do idoso em um Centro de Especialidades Médicas no Pará, no período de 2014 a 2016, correlacionando a prevalência de polifarmácia de acordo com o gênero e a faixa etária dos pacientes estudados.

\section{MÉTODOS}

O estudo realizado foi observacional quantitativo, descritivo de análise exploratória documental, utilizando dados dos prontuários de um ambulatório, em Belém-PA. Portanto, não houve qualquer contato direto com os pacientes, mas sim, com os seus registros existentes no arquivo médico, por esse motivo não se fez necessário a elaboração do Termo de Consentimento Livre e Esclarecido (TCLE).

As informações coletadas dos prontuários dos pacientes idosos foram utilizadas exclusivamente para fins de elaboração do presente trabalho, sendo garantida a privacidade da identificação dos prontuários a serem utilizados no estudo.

A amostra foi formada por um total de 241 prontuários de pessoas idosas atendidas no período de janeiro de 2014 a dezembro de 2016. Foram estudadas nesse projeto, somente as primeiras consultas registradas nos prontuários dos pacientes atendidos pela primeira vez no ambulatório.

Foram excluídos da pesquisa os prontuários de pessoas idosas atendidas em outros ambulatórios e os prontuários nos quais não existia registro de prescrição medicamentosa na primeira consulta. Também foram excluídos os prontuários de pacientes que iniciaram atendimento no ambulatório antes de janeiro de 2014 . $\mathrm{Na}$ análise dos dados foram excluídos os pacientes com meia idade (45 a 59 anos).

Foram utilizados dados como: idade, sexo, religião, estado civil, situação trabalhista, escolaridade, doenças ou diagnósticos prévios e medicamentos.

Os medicamentos prescritos foram comparados com a lista dos fármacos pertencentes ao programa Farmácia Popular para análise do acesso aos medicamentos. Para o presente estudo a classificação da faixa etária dos idosos utilizada foi a estabelecida pela Organização Mundial de Saúde (OMS): idoso (60-74 anos), ancião (75-89 anos) e velhice extrema ( $\geq 90$ anos). Para a tabulação de dados, foi utilizado o conceito de polifarmácia como uso de cinco ou mais medicamentos.

Uma vez inclusos, os dados foram consolidados sob a forma de números absolutos e ou relativos e apresentados sob a forma de gráficos e tabelas e analisadas com a estatística descritiva e Qui-Quadrado de Aderência, com auxílio do programa BioEstat 5.0. Foi aceito como diferença estatisticamente significativa pvalor 0,05 .

Este trabalho foi iniciado após submissão e aprovação pelo Comitê de Ética em Pesquisa em Seres Humanos do Centro Universitário do Estado do Pará (CEP-CESUPA) sob o processo de número 908.225 em 10 de dezembro de 2014. 


\section{RESULTADOS}

De 249 prontuários analisados, 8 foram excluídos por não possuírem o registro da idade e/ou data do nascimento do paciente. Houve comprometimento na coleta de dados dos prontuários visto que a parte inicial que abrange dados sóciodemográficos estava em branco em grande número de prontuários.

Houve predomínio do sexo feminino $(69,1 \%)$ e de idosos (60 a 74 anos) $(60,2 \%)$ com diferença estatística significante em relação às demais faixas de idade analisadas (Tabela 1).

Tabela 1 - Distribuição, segundo sexo e faixa etária dos idosos atendidos no ambulatório de Saúde do Idoso do Centro de Especialidades Médicas do Centro Universitário do Estado do Pará, no período de 2014 a 2016.

\begin{tabular}{lccc}
\hline Características & $\mathbf{N}=\mathbf{2 3 6}$ & $\%$ & p-valor \\
\hline Sexo & 73 & & \\
Masculino & 163 & 30,9 & \\
Feminino & & 69,1 & $<0,0001$ \\
Faixa Etária & 142 & 60,2 & $<0,0001$ \\
Idoso (60-74 anos) & 89 & 37.7 & \\
Ancião (75-90 anos) & 5 & 2.1 & \\
Velhice extrema (>90 anos) & &
\end{tabular}

Fonte: Maués CR, Fernandez MM, Nunes QP, et al., 2017.

* p-valor-(Teste Qui-Quadrado de Aderência).

$\mathrm{Na}$ tabela 2 observa-se que, excluindo-se os registros ignorados houve diferença estatística significante relacionada à maior prevalência de católicos $(13,1 \%)$, casados $(40,7 \%)$, aposentados $(32,6 \%)$ e com primeiro grau de escolaridade $(22,0 \%)$.

Tabela 2- Distribuição, segundo algumas variáveis sócio-demográficas dos idosos atendidos no ambulatório de Saúde do Idoso do Centro de Especialidades Médicas do Centro Universitário do Estado do Pará, no período de 2014 a 2016.

\begin{tabular}{|c|c|c|c|c|c|}
\hline \multirow[b]{2}{*}{ Características } & \multicolumn{3}{|c|}{ Faixa Etária } & \multirow[b]{2}{*}{$\begin{array}{l}\text { Total } \\
\mathrm{N}=236\end{array}$} & \multirow[b]{2}{*}{$\%$} \\
\hline & $\begin{array}{l}\text { Idoso } \\
\mathrm{N}=142\end{array}$ & $\begin{array}{l}\text { Ancião } \\
\mathrm{N}=89\end{array}$ & $\begin{array}{l}\text { Velhice extrema } \\
\mathrm{N}=5\end{array}$ & & \\
\hline \multicolumn{6}{|l|}{ Religião } \\
\hline Católico & 21 & 10 & - & 31 & $13,1^{*}$ \\
\hline Evangélico & 16 & 5 & - & 21 & 8,9 \\
\hline Outras & 1 & 1 & - & 2 & 0,8 \\
\hline Ignorado & 104 & 73 & 5 & 182 & 77,1 \\
\hline \multicolumn{6}{|l|}{ Estado Civil } \\
\hline Casado & 58 & 37 & 1 & 96 & $40,7^{\star}$ \\
\hline Solteiro & 45 & 17 & 1 & 63 & 26,7 \\
\hline Separado/divorciado & 21 & 3 & - & 24 & 10,2 \\
\hline Viúvo & 12 & 24 & 3 & 39 & 16,5 \\
\hline Ignorado & 6 & 8 & - & 14 & 5,9 \\
\hline \multicolumn{6}{|l|}{ Situação Trabalhista } \\
\hline Empregado & 20 & 6 & - & 26 & 11,0 \\
\hline Desempregado & 8 & 3 & - & 11 & 4,7 \\
\hline Aposentado & 43 & 33 & 1 & 77 & $32,6^{*}$ \\
\hline Ignorado & 71 & 47 & 4 & 122 & 51,7 \\
\hline \multicolumn{6}{|l|}{ Escolaridade } \\
\hline Analfabeto & 8 & 8 & - & 16 & 6,8 \\
\hline Primeiro grau & 33 & 19 & - & 52 & $22,0^{*}$ \\
\hline Segundo grau & 11 & 3 & - & 14 & 5,9 \\
\hline Terceiro grau & 1 & 1 & - & 2 & 0,8 \\
\hline Ignorado & 89 & 58 & 5 & 152 & 64,4 \\
\hline
\end{tabular}

Fonte: Maués CR, Fernandez MM, Nunes QP, et al., 2017. * $p$-valor $<0,0001$ (Teste Qui-Quadrado de Aderência). Nota: Idoso (60 a 74 anos); Ancião ( 75 a 89 anos) e Velhice extrema ( $\geq 90$ anos). 
Na distribuição das doenças ou diagnósticos prévios ou de base foi incluído mais de um registro para cada idoso no estudo e excluído os pacientes com meia idade (45 a 59 anos). Predominaram, dentre as doenças do aparelho circulatório (73,0\%), a hipertensão $(75,6 \%)$ e nas doenças nutricionais, endócrinas e metabólicas $(36,9 \%)$ o diabetes $(49,4 \%)$ (Tabela 3$)$.

Tabela 3 - Distribuição das doenças segundo faixa etária, dos idosos atendidos no ambulatório de Saúde do Idoso do Centro de Especialidades Médicas do Centro Universitário do Estado do Pará, no período de 2014 a 2016.

\begin{tabular}{|c|c|c|c|c|c|}
\hline $\begin{array}{l}\text { Doenças } \\
\text { Referidas }\end{array}$ & Idoso & Ancião & $\begin{array}{l}\text { Velhice } \\
\text { extrema }\end{array}$ & Total & $\%$ \\
\hline Doenças do aparelho circulatório & 84 & 86 & 5 & 175 & 73,0 \\
\hline Hipertensão & 74 & 55 & 4 & 133 & 75,6 \\
\hline \multicolumn{6}{|l|}{ Pessoas em contato com serviços de saúde } \\
\hline para procedimentos e cuidados específicos & 90 & 50 & 4 & 144 & 60,2 \\
\hline Cirurgias & 71 & 36 & 2 & 109 & 75,9 \\
\hline Internações & 19 & 14 & 2 & 35 & 24,1 \\
\hline Doenças nutricionais, endócrinas e & & & & & \\
\hline metabólicas & 49 & 35 & 3 & 87 & 36,9 \\
\hline Diabetes & 24 & 15 & 3 & 42 & 49,4 \\
\hline Dislipidemia & 22 & 15 & & 37 & 41,6 \\
\hline Doenças do aparelho digestivo & 11 & 11 & 0 & 22 & 10,0 \\
\hline \multicolumn{6}{|l|}{ Doenças do sistema osteomuscular e do } \\
\hline tecido conjuntivo & 4 & 11 & & 15 & 7,5 \\
\hline Transtornos mentais e comportamentais & 9 & 5 & 1 & 15 & 6,2 \\
\hline Doenças do olho e anexos & 11 & 3 & 1 & 15 & 6,2 \\
\hline Doenças do aparelho respiratório & 26 & 11 & 1 & 38 & 16,2 \\
\hline Neoplasias & 4 & 4 & 1 & 9 & 3,7 \\
\hline Doenças do aparelho geniturinário & 4 & 7 & 1 & 12 & 5,0 \\
\hline
\end{tabular}

Fonte: Maués CR, Fernandez MM, Nunes QP, et al., 2017.

Nota: Idoso (60 a 74 anos); Ancião (75 a 89 anos) e Velhice extrema ( $\geq 90$ anos).

Na Tabela 4, observa-se o registro das doenças relacionadas ao número de medicamentos utilizados pelos idosos. De acordo com o teste estatístico utilizado, não há diferença estatística significante $(p=0,5788)$ entre o número de doenças e o número de medicamentos utilizados pelos pacientes idosos.

Tabela 4- Distribuição, segundo doenças e número de medicamentos registrados, dos idosos atendidos no ambulatório de Saúde do Idoso do Centro de Especialidades Médicas do Centro Universitário do Estado do Pará, no período de 2014 a 2016.

\begin{tabular}{llllllllll}
\hline \multirow{2}{*}{ Número de doenças } & \multicolumn{1}{l}{ Número de medicamentos utilizados } \\
\cline { 2 - 10 } & 0 & 1 & 2 & 3 & 4 & 5 & 6 & Total & $\%$ \\
\hline 1 & 29 & 24 & 13 & 10 & 1 & 1 & 2 & 80 & $33,2^{*}$ \\
2 & 14 & 13 & 12 & 19 & 5 & 9 & 1 & 73 & 30,3 \\
3 & 4 & 9 & 8 & 4 & 5 & 5 & 7 & 42 & 17,4 \\
4 & 2 & 4 & 5 & 1 & 5 & 6 & 2 & 25 & 10,4 \\
5 & 3 & 1 & 1 & 1 & 1 & 1 & 1 & 9 & 3,7 \\
6 & 1 & 1 & 1 & 1 & 1 & 2 & 0 & 7 & 2,9 \\
7 & 0 & 0 & 1 & 0 & 0 & 2 & 2 & 5 & 2,1 \\
\hline Total & $53^{*}$ & 52 & 41 & 36 & 18 & 26 & 15 & 241 & 100,0 \\
$\%$ & 22,0 & 21,6 & 17,0 & 14,9 & 7,5 & 10,8 & 6,2 & 100,0 & \\
\hline
\end{tabular}

Fonte: Maués CR, Fernandez MM, Nunes QP, et al., 2017.

${ }^{*}$-valor $=0,5788$ (Teste Q-Cochran). 
Na Tabela 5 observa-se que os ansiolíticos, anti-histamínicos e os antidiabéticos (insulina) prescritos aos pacientes tem $100,0 \%$ de disponibilidade no Programa da Farmácia Popular. No entanto a maioria dos medicamentos prescritos não é dispensada por esse programa. Os anti-hipertensivos são os medicamentos mais prescritos (214) com $81,3 \%$ de disponibilidade no programa da Farmácia Popular, seguido dos hipoglicemiantes orais (55) e antiagregante plaquetário (31).

Tabela 5 - Distribuição segundo a disponibilidade pela Farmácia Popular do Brasil dos medicamentos prescritos para os idosos atendidos no ambulatório de Saúde do Idoso do Centro de Especialidades Médicas do Centro Universitário do Estado do Pará, no período de 2014 a 2016.

\begin{tabular}{|c|c|c|}
\hline Classe & $\begin{array}{l}\text { Frequência de } \\
\text { prescrição }\end{array}$ & $\begin{array}{l}\text { Proporção (\%) dos medicamentos } \\
\text { disponíveis na farmácia popular }\end{array}$ \\
\hline Ansiolíticos & 7 & 100,0 \\
\hline Anti-histamínicos & 1 & 100,0 \\
\hline Antidiabético (Insulina) & 5 & 100,0 \\
\hline Antiagregante plaquetário & 31 & 93,5 \\
\hline Hipoglicemiantes orais & 55 & 92,7 \\
\hline Hipolipemiante & 29 & 89,0 \\
\hline Anti-hipertensivos & 214 & 81,3 \\
\hline Corticoide & 4 & 75,0 \\
\hline Protetores gástricos & 19 & 68,4 \\
\hline Anticonvulsivante & 3 & 66,6 \\
\hline Bifosfonado & 8 & 62,5 \\
\hline Antiarrítmico & 7 & 57,1 \\
\hline Antidepressivos & 14 & 21,4 \\
\hline Suplementos & 23 & 8,7 \\
\hline Analgésicos & 14 & 7,1 \\
\hline Antiinflamatório & 18 & 5,5 \\
\hline Agonista dopaminérgico & 1 & 0,0 \\
\hline Antineurálgico & 1 & 0,0 \\
\hline Anti reumáticos & 2 & 0,0 \\
\hline Anticolinesterásico & 3 & 0,0 \\
\hline Antiespasmódicos & 5 & 0,0 \\
\hline Antiparkinsoniano & 2 & 0,0 \\
\hline Antipsicótico & 4 & 0,0 \\
\hline Antivertiginosos & 7 & 0,0 \\
\hline Broncodilatadores & 4 & 0,0 \\
\hline Estimulante coginitivo & 1 & 0,0 \\
\hline Hormônios & 7 & 0,0 \\
\hline Inibidor prostático & 3 & 0,0 \\
\hline Laxantes & 2 & 0,0 \\
\hline Relaxantes musculares & 3 & 0,0 \\
\hline Supres horm. crescimento & 2 & 0,0 \\
\hline Supressor tireoideo & 3 & 0,0 \\
\hline Vasotônicos & 11 & 0,0 \\
\hline Outros & 8 & 0,0 \\
\hline
\end{tabular}

Fonte: Maués CR, Fernandez MM, Nunes QP, et al., 2017.

O número de pacientes idosos (60 a 74 anos) que utilizam um ou dois medicamentos corresponde, aproximadamente, ao dobro dos anciãos ( 75 a 89 anos). No entanto essa relação se aproxima no uso de três e cinco e mais medicamentos e se inverte quando o uso é de quatro medicamentos. Essa inversão é representada pela diferença estatística significante $(p=0,0473)$ (Tabela 6). 
Tabela 6- Distribuição, segundo o número de medicamentos registrados e a faixa etária dos idosos atendidos no ambulatório de Saúde do Idoso do Centro de Especialidades Médicas do Centro Universitário do Estado do Pará, no período de 2014 a 2016.

\begin{tabular}{|c|c|c|c|c|}
\hline \multirow{2}{*}{$\begin{array}{c}\text { Número de } \\
\text { Medicamentos }\end{array}$} & \multicolumn{3}{|c|}{ Faixa Etária } & \multirow[b]{2}{*}{ Total } \\
\hline & 60 a 74 anos & 75 a 89 anos & $\geq 90$ anos & \\
\hline 1 & 35 & 16 & - & 51 \\
\hline 2 & 24 & 13 & 2 & 39 \\
\hline 3 & 18 & 17 & 1 & 36 \\
\hline 4 & 5 & 12 & - & 17 \\
\hline $5 e+$ & 18 & 22 & 1 & 41 \\
\hline Ignorado & 42 & 9 & 1 & 52 \\
\hline Total & 142 & 89 & 5 & 236 \\
\hline
\end{tabular}

Fonte: Maués CR, Fernandez MM, Nunes QP, et al., 2017.

${ }^{*} \mathrm{p}$-valor $=0,0473$ (Teste Q-Cochran).

\section{DISCUSSÃo}

No contexto atual e crescente do desenvolvimento técnico-cientifico, através dos novos conhecimentos acerca da fisiopatologia e de novas abordagens, houve a extensão das opções de tratamento e do acesso da população aos fármacos. Com isso, ganhou espaço um dos problemas dentro da pratica geriátrica: a iatrogenia. É considerado como afecção iatrogênica, aquela que se relaciona diretamente com a intervenção feita pelo médico e/ou seus auxiliares, sendo correta ou errada, com justificativa ou não, porém da qual resulta algum tipo de consequência prejudicial para a saúde do paciente. É valido destacar que os pacientes hospitalizados são os mais propensos a esse tipo de prática (CARVALHO-FILHO ET, et al., 1998).

Dos 236 pacientes estudados, 163 eram do sexo feminino $(69,1 \%)$ contra 73 do sexo masculino $(30,9 \%)$, sendo possível observar um predomínio do sexo feminino nessa população. Isso pode ser explicado devido ao fato de que as transformações demográficas que vem ocorrendo nas últimas décadas do século XX, tais como mudança nos padrões culturais, expansão da escolaridade e 0 ingresso nas universidades, transformando inclusive o papel da mulher dentro do contexto doméstico familiar, se mostram plenamente observados na amostra estudada (BRUSCHINI MCA, 2015).

Considera- se que, quanto mais velho for o contingente analisado, maior será a proporção de mulheres, dada as hipóteses de que são menos expostas a riscos, acidentes de trânsito, domésticos e de trabalho, homicídios e suicídios, consomem menos tabaco e álcool e fazem uso mais frequente dos serviços de saúde (CAMARANO AA, 2002; VERAS RP, et al., 2001).

Os indivíduos do estudo foram divididos em faixas etárias, sendo a mais prevalente a de idosos, com 152 pacientes entre 60 e 74 anos $(60,2 \%)$, o que diverge de outro estudo, que também dividiu seu estudo em faixas etárias e encontrou a maioria de sua amostra (28,6\%) entre $72-77$ anos (ANDRADE AINPA, MARTINS RML, 2014).

O crescimento da população de idosos é um fenômeno mundial e está ocorrendo em nível sem precedentes. A Organização Mundial da Saúde (OMS) considera idosas as pessoas com mais de 65 anos. Este referencial, entretanto, é válido para habitantes de países desenvolvidos. Nos países em desenvolvimento, como o Brasil, velhice começa aos 60 anos. Esse segmento da população, pelo próprio processo de envelhecimento, apresenta maior prevalência de doenças crônico-degenerativas (BARBOSA BR, et al., 2014; NAKATANI AYK, et al., 2009).

A longevidade acarreta consequências significativas para a qualidade de vida, pois ocorrem inúmeras modificações, seja em âmbito individual ou coletivo. Há aumento da susceptibilidade para um extenso leque de doenças, além do agravamento de doenças prévias, também ocorrem perdas afetivas, as quais podem gerar transtornos psiquiátricos, dependendo do suporte que tal idoso obtiver (ALVES LC, et al., 2003).

A baixa de escolaridade pode interferir no dia-a-dia do idoso, ocasionando dificuldades ao manusear os medicamentos, seguir dietas ou prescrições. Provavelmente reconhecerão seus medicamentos somente pela 
embalagem, cor ou formato dos comprimidos. Nessa forma insuficiente, pode ocorrer troca de medicação, que trará prejuízos ao idoso e maiores riscos à sua saúde, sendo necessária uma orientação redobrada dos profissionais para com seus acompanhantes ou cuidadores, a fim de ensiná-los a prevenir possíveis enganos (GARCIA ESS, SAINTRAIN MVL, 2009).

Juntamente com o aumento da idade populacional, que já é uma realidade no país, aumentam os índices de morbidade, trazendo concomitantemente 0 aumento das doenças crônicas de início silencioso, que demandam um alto custo com cuidados de saúde, além de poderem ser extremamente incapacitantes para os idosos (ALVES LC, et al., 2008).

A doença mais prevalente encontrada foi hipertensão arterial $(75,6 \%)$, contida no grupo das doenças do aparelho circulatório, seguida de diabetes $(49,4 \%)$, do grupo de doenças nutricionais, endócrinas e metabólicas, e, em terceira posição, os distúrbios respiratórios (16.2\%). Estes achados foram corroborados por um estudo que utilizou dados da pesquisa nacional de amostra por domicílio (PNAD) que encontrou 16.555 idosos hipertensos, seguido de problemas na coluna (12.501) e artrite/reumatismo (9.460) diferindo, portanto, quanto ao segundo e terceiro achados (ALVES LC, et al., 2008).

As doenças do aparelho circulatório se destacam ocupando o primeiro lugar dentre as causas de morte nos idosos. As que mais evoluem para o óbito são as moléstias cerebrovasculares e isquêmicas do coração, todas relacionadas à hipertensão arterial. Para outros autores também merecem destaque as doenças com acometimento pulmonar, como DPOC, câncer de pulmão e pneumonia (COSTA MFFL, et al., 2000; CHAIMOWICZ F, 2013).

O diabetes foi a segunda morbidade mais prevalente observada, com 118 pacientes (49\%) acometidos por essa patologia. Essa patologia tem grande importância relativa, se destacando como causa de óbito principalmente no sexo feminino. $O$ diabetes mellitus tipo 2 se destaca pela sua alta prevalência no mundo, sendo maior entre os idosos. É válido lembrar que ele é um dos principais fatores de risco para doença cardiovascular e cerebrovascular (CHAIMOWICZ F, 2013; SARTORELLI DS e FRANCO LJ, 2003).

Outro ponto muito relevante a ser avaliado é a propensão do idoso à instabilidade postural, que propicia o maior risco de quedas se relacionando a elevados índices de hospitalizações precoces e morbimortalidade. Concordando com essa relação, o estudo de prevalência de quedas em idosos e fatores associados encontrou uma prevalência de $34,8 \%$ de quedas em idosos (ALVES LC, et al., 2008; SIQUEIRA FV, et al., 2007).

Participar de atividades programadas em grupos traz aos idosos benefícios de realizar tarefas nas quais ele está apto a exercer sua autonomia, dentro de um contexto onde as pessoas ao redor possuem a mesma vontade de envelhecer ativamente. Com isso, eles interagem, mantêm-se socialmente ativos e elevam a autoestima, recebendo estímulos que vão além do físico, como também do intelectual (PEREIRA GR, 2005).

Em relação à polifarmácia, foi observado que a classe mais prescrita foi a dos anti-hipertensivos, seguida por hipoglicemiantes orais e dos antiagregantes plaquetários. Tal achado discorda de um estudo realizado no abrigo São Vicente em Cascavel-PR onde foi demonstrado que as classes mais prescritas foi a dos psicotrópicos, seguida por anti-hipertensivos e pelos agentes antiulcerosos (FLEMING I, GOETTEN LF, 2005).

De acordo com a literatura, dentre os remédios mais prescritos na população idosa, estão aqueles com ação sobre o sistema cardiovascular (55\%), uma vez que as doenças cardiovasculares são as principais causadoras de óbito nessa população. Esse dado concorda com o presente achado de $73 \%$ de idosos acometidos por doenças cardiovasculares (BJERRUM L, et al., 1997).

A elevada prevalência de comorbidades na população idosa justifica a maior tomada de medicamentos nessa faixa etária. Os medicamentos mais utilizados seguem a mesma distribuição das principais doenças crônico-degenerativas mais prevalentes na população, tais como medicamentos para patologias cardiovasculares, seguidos por psicotrópicos, como neurolépticos, antidepressivos, benzodiazepínicos e anticonvulsivantes. Essa distribuição não foi observada no presente estudo, pois segundo os dados 
analisados, o número de doenças não guarda correlação com o número de medicamentos prescritos, podendo ser observado na tabela 6 (GALVÃO C, 2006).

Em relação ao uso de medicamentos por faixa etária, foi possível estabelecer através desse estudo, que a polifarmácia é mais frequente nos anciãos $(9,3 \%)$ quando comparada aos idosos $(7,6 \%)$, concordando com outro estudo, que afirma que o uso de medicamentos é maior na faixa etária de 70 anos em diante $(87,3 \%)$ quando comparado à polifarmácia em pessoas da faixa etária de 60-69 anos (78,8\%) (SILVA AL, et al., 2012) .

Casuísticas ambulatoriais notam possíveis associações da polifarmácia com sexo feminino, idade avançada, maior número de consultas no último ano, maior renda familiar, número de comorbidades, morar sozinho e ausência de déficit cognitivo (demência) (COELHO FJM, et al., 2004; LOYOLA FAI, et al., 2008).

Há uma relação exponencial entre polifarmácia e a probabilidade de reações adversas, interações medicamentosas e medicamentos inapropriados para idosos, podendo intervir em processos como a qualidade do sono, nutrição e risco de quedas (PASSARELLI MCG, 2008; NEIKRUG AB e ANCOLI-ISRAEL S, 2009; AMER MS, et al., 2009).

\section{CONCLUSÃO}

No presente estudo, foi identificado o predomínio de mulheres na faixa etária de 60-74 anos. Foi observado maior prevalência da religião católica, de casados, aposentados e com escolaridade do primeiro grau. As doenças mais prevalentes foram hipertensão, seguida de diabetes e distúrbios respiratórios. Houve baixa prevalência de etilismo e tabagismo, assim como de atividade física. Foi observado que as classes de medicamentos mais prescritas foram anti-hipertensivos, antidiabéticos orais e antiagregante plaquetários. A maioria dos prontuários analisados continha apenas um diagnóstico de base e sem uso de medicação contínua, porém, entre aqueles com duas ou mais doenças, a polifarmácia foi frequente, com uso de três ou mais medicamentos concomitantes. Embora a polifarmácia seja um tema preocupante na prescrição para os pacientes idosos, observou-se que parece haver uma relação direta entre o número de medicamentos e as idades mais avançadas.

\section{REFERÊNCIAS}

1. CIOSAK SI, et al. Senescência e senilidade: o novo paradigma na atenção primaria a saúde. Rev. esc. enferm. USP. $2011 ; 45(2): 1763-68$.

2. PEREIRA LRL, et al. Avaliação da utilização de medicamentos em pacientes idosos por meio de conceitos fármaco epidemiologia e fármaco vigilância. Ciência \& saúde coletiva. 2004; 9(2):479-81.

3. ARAÚJO ENP, LOPES RGC. Instituições de Longa Permanência para Idosos: possibilidades contemporâneas de moradia. Revista Kairós Gerontologia. 2010; 13: 45-60.

4. MOSEGUI GBG, et al. Avaliação da qualidade do uso de medicamentos em idosos. Rev. Saúde Pública. 2005; 33(5): 437-44.

5. ROZENFELD S. Prevalência, fatores associados e mau uso de medicamentos entre os idosos: uma revisão. Caderno de Saúde Pública. 2003; 19(3):717-24.

6.ACURCIO FA, et al. Complexidade do regime terapêutico prescrito para idosos. Rev. Assoc. Med. Bras. 2009; 55(4): 468-74.

7.CORRER CJ, et al. Riscos de problemas relacionados com medicamentos em pacientes de uma instituição geriátrica. Revista Brasileira de Ciências Farmacêuticas. 2007; 43(1).

8. MARIN MJS, et al. Caracterização do uso de medicamentos entre idosos de uma unidade do Programa Saúde da Família. Cad. Saúde Pública. 2008; 24(7):1545-55

9. AMER, MS, et al. Malnutrition and its risk factors in nursing home residents in Cairo. J Am Geriatr Soc 2009.

10. SCHRAMM JMA, et al. Transição epidemiológica e o estudo de carga de doença no Brasil, Ciência \& Saúde Coletiva. $2004 ; 9(4): 897-908$.

11. FRENK J, et al. La transición epidemiológica en América Latina. Boletín de la Oficina Sanitaria Panamericana. 1989; 111(6):485-96.

12. ROMANO-LIEBER NS, et al. Revisão dos estudos de intervenção do farmacêutico no uso de medicamentos por pacientes idosos. Cad. Saúde Pública. 2002; 18(6), p.1499-1507. 
13. CARVALHO-FILHO ET, et al. latrogenia em pacientes idosos hospitalizados. Rev. Saúde Pública.1998; 32(1):36-42.

14. BRUSCHINI MCA. Trabalho e gênero no brasil nos últimos 10 anos. Cadernos de pesquisa. 2015; 37(132): 537-72.

15. CAMARANO AA. Envelhecimento da população brasileira: uma contribuição demográfica, orçamento e gestão. Rio de Janeiro: IPEA/Ministério do Planejamento; 2002.

16. VERAS RP, et al. Desafios e conquistas advindas da longevidade da população: o setor saúde e suas necessárias transformações. In: Veras RP, organizadores. Velhice numa perspectiva saudável. Rio de Janeiro: UnATI-UERJ; 2001. p. 11-32.

17. ANDRADE AINPA, MARTINS RML. Funcionalidade Familiar e Qualidade de vida dos idosos. Millenium. 2014; 40: 185-99.

18. BARBOSA BR, et al. Avaliação da capacidade funcional dos idosos e fatores associados à incapacidade Ciência \& Saúde Coletiva. 2014; 19(8):3317-25.

19. NAKATANI AYK, et al. Capacidade funcional em idosos na comunidade e propostas de intervenções pela equipe de saúde. Rev Eletrônica Enfermagem. 2009; 11(1):144-50.

20. ALVES LC, et al. Health profile of the elderly in Brazil: analysis of the 2003 National Household Sample Survey using the Grade of Membership method. Cad Saúde Pública. 2008; 24(3):552-46.

21. GARCIA ESS, SAINTRAIN MVL. Perfil epidemiológico de uma população idosa atendida pelo programa saúde da família. Rev. enferm. UERJ, Rio de Janeiro. 2009; 17(1):18-23.

22. COSTA MFFL, et al. Diagnóstico da situação de saúde da população brasileira: um estudo da mortalidade e das internações hospitalares públicas. Informe Epidemiológico do SUS. 2000; 9(1): 23-41.

23. CHAIMOWICZ F. Saúde do Idoso, 2 Ed, 2013.

24. SARTORELLI DS, FRANCO LJ. Tendências do diabetes mellitus no Brasil: o papel da transição nutricional. Cad Saúde Pública. 2003; 19 Suppl 1:29-36.

25. SIQUEIRA FV, et al. Prevalência de quedas em idosos e fatores associados. Rev Saúde Pública. 2007; 41(5):74956.

26. PEREIRA GR. A influência dos grupos de convivência na qualidade de vida dos idosos no município de Garopaba. Trabalho de conclusão de curso de serviço social, UFSC, Florianópolis, 2005.

27. FLEMING I, GOETTEN LF. Medicamentos mais utilizados pelos idosos: implicações para a enfermagem. Arq. Ciênc. Saúde Unipar. 2005; 9(2):p.121-28.

28. BJERRUM L, et al. Methods for estimating the occurence of polypharmacy by means of prescription database. Eur $\mathrm{J}$ Clin Pharmacol. 1997.

29. GALVÃO C. Idoso polimedicado - Estratégias para melhorar a prescrição. Revista Portuguesa de Clinica Geral. 2006; Rev Bras Geriatria e Gerontologia. 2016; 22(6): 747-52.

30. SILVA AL, et al. Utilização de medicamentos por idosos brasileiros, de acordo com a faixa etária: um inquérito postal. Cad. Saúde Pública. 2012; 28(6):1033-45.

31. COELHO FJM, et al. Perfil de utilização de medicamentos por idosos em área urbana do Nordeste do Brasil. Rev Saúde Pública. 2004; Rev Bras Geriatria e Gerontologia. 2016; 8(1): 9-20.

32. LOYOLA Al, et al. Influência da renda na associação entre disfunção cognitiva e polifarmácia: Projeto Bambuí. Cad Saude Publica. 2008; 42(1): 89-99.

33. PASSARELLI MCG, GORZONI ML. latrogenia: Reações adversas a medicamentos. In: Jacob Filho W, Gorzoni ML. Geriatria e Gerontologia: o que todos deviam saber. São Paulo: Roca; 2008

34. NEIKRUG AB, ANCOLI-ISRAEL S. Sleep Disorders in the Older Adult: a mini-review. Gerontology 2009. 